\title{
A STUDY OF CHEMICALLY REACTIVE SPECIES AND THERMAL RADIATION EFFECTS ON AN UNSTEADY MHD FREE CONVECTION FLOW THROUGH A POROUS MEDIUM PAST A FLAT PLATE WITH RAMPED WALL TEMPERATURE
}

\author{
K.K. PANDIT ${ }^{*}$ \\ Department of Mathematics, Gauhati University \\ Guwahati, 781014, Assam, INDIA \\ E-mail: kamalesh.pandit@yahoo.co.in \\ D. SARMA \\ Department of Mathematics, Cotton College \\ Guwahati 781001, Assam, INDIA \\ E-mail: dipaksarma11@yahoo.com \\ S.I. SINGH \\ Department of Mathematics, SonapurCollege \\ Guwahati, 782402, Assam, INDIA \\ E-mail: sinamisingh@gmail.com
}

\begin{abstract}
An investigation of the effects of a chemical reaction and thermal radiation on unsteady MHD free convection heat and mass transfer flow of an electrically conducting, viscous, incompressible fluid past a vertical infinite flat plate embedded in a porous medium is carried out. The flow is induced by a general time-dependent movement of the vertical plate, and the cases of ramped temperature and isothermal plates are studied. An exact solution of the governing equations is obtained in closed form by the Laplace Transform technique. Some applications of practical interest for different types of plate motions are discussed. The numerical values of fluid velocity, temperature and species concentration are displayed graphically whereas the numerical values of skin friction, Nusselt number and Sherwood number are presented in a tabular form for various values of pertinent flow parameters for both ramped temperature and isothermal plates.
\end{abstract}

Key words: free convection, chemical reaction, thermal radiation, MHD, porous medium, ramped temperature.

\section{Introduction}

In recent years, the flows of fluid through porous media have been of principal interest because they are quite prevalent in nature. Such flows have attracted the attention of a number of scholars due to their application in many branches of science and technology, viz., in the field of agriculture engineering to study the underground water resources, seepage of water in river-beds, in petroleum technology to study the movement of natural gas, oil and reservoirs, in chemical engineering for filtration and purification processes. The convection problem in porous media has also important applications in geothermal reservoirs and geothermal energy extractions. A comprehensive review of the studies of the convective heat transfer mechanism through porous media has been made by Nield and Bejan [1]. Hiremath and Patil [2] studied the effect of free convection currents on the oscillatory flow through a porous medium which is bounded by a vertical plane surface of constant temperature. Combined heat and mass transfer flows are of considerable importance in many applications. These include transport phenomena in heating and cooling processes in

\footnotetext{
${ }^{*}$ To whom correspondence should be addressed
} 
semi-conductor electronics, power transformer electronics, absorption reactors, solar energy system, binary diffusion system and polymer processing in the plastic industry.

Free convection flow involving coupled heat and mass transfer occurs frequently in nature. It occurs not only due to temperature differences, but also due to concentration differences or a combination of these two, for example, in atmospheric flows there exist differences in the $\mathrm{H}_{2} \mathrm{O}$ concentration. A few representative fields of interest in which combined heat and mass transfer plays an important role is designing of chemical processing equipment, distribution of temperature, formation and dispersion of fog and moisture over agricultural fields and groves of fruit trees, environmental pollution and crop damage due to freezing.

The effect of thermal radiation and chemical reaction on MHD boundary layer flow has become important in several industrial, scientific and engineering fields. There are many transport processes that are governed by the combined action of buoyancy forces due to both thermal and mass diffusion in the presence of chemical reactions. These processes are observed in nuclear reactor safety and combustion systems, solar collectors. From the point of view of applications, Muthucumaraswamy et al. [3] investigated the effect of an unsteady flow past an exponentially accelerated infinite isothermal vertical plate with variable mass diffusion in the presence of a first-order homogeneous chemical reaction. Das et al. [4] studied the effect of the firstorder homogeneous chemical reaction of an unsteady flow past a vertical plate with constant heat and mass transfer. In view of this, Suneetha et al. [5] investigated the effects of thermal radiation on the natural convective heat and mass transfer of a viscous incompressible gray absorbing-emitting fluid flowing past an impulsively started moving vertical plate with viscous dissipation. Sattar and Kalim [6] studied the unsteady free convection interaction with thermal radiation in a boundary layer flow past a vertical porous plate. Aydin and Kaya [7] investigated the effect of thermal radiation on MHD mixed convection flow about a permeable vertical plate. Many researchers such as Arpaci [8], Cess [9], Cheng and Ozisiki [10], Raptis [11], Hossain and Takhar $[12 ; 13]$ investigated the interaction of thermal radiation and free convection for different geometries, by considering the flow to be steady. The unsteady flow past a moving vertical plate in the presence of free convection and radiation weas studied by Das et al. [14]. Finite difference solutions of an MHD mixed convection flow with heat generation and chemical reaction were studied by Ahmed et al. [15]. Seth et al. [16] investigated the effects of Hall current, radiation and rotation on natural convection heat and mass transfer flow past a moving vertical plate. Cookey et al. [17] analyzed the influence of viscous dissipation and radiation on an unsteady MHD free convection flow past an infinite heated vertical plate in a porous medium with time-dependent suction. Suneetha et al. [18] studied the effects of thermal radiation on an MHD free convection flow past an impulsively started vertical plate with variable surface temperature and concentration. Mahmoud [19] studied the effect of thermal radiation on an unsteady MHD free convection flow past a vertical plate with temperature dependent viscosity. Seth et al. [20] studied the effects of thermal radiation and rotation on an unsteady hydromagnetic free convection flow past an impulsively moving vertical plate with ramped temperature in a porous medium.

Hydro magnetic flows and heat transfer have become more important in recent years because of varied applications in agricultural engineering and petroleum industries. Recently, considerable attention has also been focussed on new applications of magneto hydrodynamics (MHD) and heat transfer. Melt refining involves magnetic field applications to control excessive heat transfer rate. Other applications of MHD heat transfer include MHD generators, plasma propulsion in astronautics, nuclear reactors and ionized-geothermal energy systems. An excellent summary of applications can be found in Hughes and Young [21]. Sacheti et al. [22] obtained an exact solution for an unsteady MHD free convection flow on an impulsively started vertical plate with constant heat flux. Takar et al. [23] analyzed the radiation effects on an MHD free convection flow past a semi-infinite vertical plate using the Runge-Kutta-Merson quadrature. Abd-El-Naby et al. [24] studied the radiation effects on an MHD unsteady free convection flow over a vertical plate with variable surface temperature. Kishore et al. [25] studied the effect of chemical reaction and radiation on an unsteady MHD free convection flow of viscous fluid past an exponentially accelerated vertical plate. ElFayez [26] studied the effect of chemical reaction on the unsteady free convection flow past an infinite vertical permeable moving plate with variable temperature. Sekhar and Reddy [27] analyzed the effect of chemical reaction on an MHD free convective oscillatory flow past a porous plate with viscous dissipation 
and heat sink. Shateyi et al. [28] studied the effect of thermal radiation and chemical reaction over an unsteady MHD flow near a stagnation point of a two-dimensional porous body with heat and mass transfer.

The purpose of the present study is to report on the effect of chemical reaction and thermal radiation on an unsteady free convective heat and mass transfer flow of a viscous, incompressible, electrically conducting and heat absorbing fluid past a vertical infinite flat plate through a porous medium. The fluid flow is induced by a general time-dependent movement of the infinite plate. This paper is the extension of the recent works of Nandkeolyar et al. [29]. The governing equations are first transformed into a set of nonsimilar equations and then solved analytically by using the Laplace transform technique and a general solution valid for any time-dependent movement of the plate is obtained. The effects of different parameters involved such as the magnetic field parameter, Schmidt number, Prandtl number, Grashof number for heat transfer and mass transfer, chemical reaction and thermal radiation on the fluid velocity, temperature and concentration distributions are plotted and discussed. Some particular cases that highlight the applications of the general solution are discussed.

\section{Mathematical formulation}

We consider an unsteady MHD free convective heat and mass transfer flow of a viscous, incompressible, electrically conducting and heat absorbing fluid along an infinite non-conducting vertical flat plate through a porous medium. The $x$-axis is along the plate in the upward direction, the $y$-axis normal to it and the $z$-axis normal to the $x y$-plane. The fluid is permeated by a uniform transverse magnetic field of strength $B_{0}$ applied along the $y$-axis. For time $t^{\prime}<0$, the stationary plate and the fluid are at same constant temperature $T_{\infty}^{\prime}$ and species concentration $C_{\infty}^{\prime}$. At time $t^{\prime}=0$, the plate begins to move with a timedependent velocity $U_{0} f\left(t^{\prime}\right)$ in its own plane along the $x$-axis and the temperature of the plate is raised or lowered to $T_{\infty}^{\prime}+\left(T_{w}^{\prime}-T_{\infty}^{\prime}\right) t^{\prime} / t_{0}$ when $t^{\prime}<t_{0}$ and thereafter, for $t^{\prime}>t_{0}$, it is maintained at a uniform temperature $T_{w}^{\prime}$. Also, for time $t^{\prime}>0$, species concentration is raised to $C_{w}^{\prime}$. The geometry of the problem is of infinite extent in $x$ and $z$ directions, and is electrically non-conducting, all physical quantities, except pressure, are functions of $y$ and $t^{\prime}$ only.

The fluid under consideration is a metallic liquid, such as mercury, whose magnetic Reynolds number is so small that the induced magnetic field produced by the fluid motion is negligible in comparison to the applied magnetic field $B=\left(0, B_{0}, 0\right)$ (Cramer and Pai [30]). Also, no external electric field is applied, so the effect of polarization of the magnetic field is negligible (Meyer [31]). With these assumptions, the governing equations for the unsteady MHD free convective heat and mass transfer flow of a viscous, incompressible, electrically conducting and heat absorbing fluid through a porous medium are as follows:

Conservation of momentum

$$
\frac{\partial u^{\prime}}{\partial t^{\prime}}=v \frac{\partial^{2} u^{\prime}}{\partial y^{\prime 2}}+g \beta\left(T^{\prime}-T_{\infty}^{\prime}\right)+g \beta^{\prime}\left(C^{\prime}-C_{\infty}^{\prime}\right)-\frac{\sigma B_{0}^{2}}{\rho} u^{\prime}-\frac{v u^{\prime}}{K^{\prime}} .
$$

Conservation of energy

$$
\frac{\partial T^{\prime}}{\partial t^{\prime}}=\frac{k}{\rho C_{p}} \frac{\partial^{2} T^{\prime}}{\partial y^{\prime 2}}-\frac{Q_{0}}{\rho C_{p}}\left(T^{\prime}-T_{\infty}^{\prime}\right)-\frac{1}{\rho C_{p}} \frac{\partial q_{r}{ }^{\prime}}{\partial y^{\prime}} .
$$

Conservation of species concentration (mass diffusion)

$$
\frac{\partial C^{\prime}}{\partial t^{\prime}}=D \frac{\partial^{2} C^{\prime}}{\partial y^{\prime 2}}-K r^{\prime}\left(C^{\prime}-C_{\infty}^{\prime}\right)
$$


where $u^{\prime}, g, \rho, \beta, \beta^{\prime}, k, C p, \sigma, \cup, D, T^{\prime}, C^{\prime}, K r^{\prime}, q_{r}{ }^{\prime}, K^{\prime}$ and $Q_{0}$ are, respectively, the fluid velocity in the $x$ direction, acceleration due to gravity, the fluid density, the volumetric coefficient of thermal expansion, the volumetric coefficient of expansion for concentration, thermal conductivity, specific heat at constant pressure, electrical conductivity, the kinematic viscosity, chemical molecular diffusivity, the temperature of the fluid, species concentration, chemical reaction parameter, radiative heat flux vector, permeability of the porous medium and the heat absorption coefficient. Assuming no slip condition between the plate and the fluid, the relevant initial boundary conditions are

$$
\begin{aligned}
& u^{\prime}=0, \quad T^{\prime}=T_{\infty}{ }^{\prime}, \quad C^{\prime}=C_{\infty}{ }^{\prime} \quad \text { for } \quad y^{\prime} \geq 0 \quad \text { and } \quad t^{\prime} \leq 0, \\
& u^{\prime}=U_{0} f\left(t^{\prime}\right), \quad C^{\prime}=C_{w}{ }^{\prime} \quad \text { at } \quad y^{\prime}=0 \text { for } t^{\prime}>0, \\
& T^{\prime}=T_{\infty}{ }^{\prime}+\left(T_{w}^{\prime \prime}-T_{\infty}^{\prime}\right) t^{\prime} / t_{0} \quad \text { at } \quad y^{\prime}=0 \text { for } 0<t^{\prime} \leq t_{0}, \\
& T^{\prime}=T_{w}{ }^{\prime} \quad \text { at } \quad y^{\prime}=0 \quad \text { for } \quad t^{\prime}>t_{0}, \\
& u^{\prime} \rightarrow 0, \quad T^{\prime} \rightarrow T_{\infty}{ }^{\prime}, \quad C^{\prime} \rightarrow C_{\infty}{ }^{\prime} \quad \text { as } \quad y^{\prime} \rightarrow \infty \text { for } \quad t^{\prime}>0 .
\end{aligned}
$$

For an optically thick fluid, in addition to emission there is also self absorption and usually the absorption co-efficient is wavelength dependent and large so we can adopt the Rosseland approximation for the radiative heat flux vector $q_{r}^{\prime}$. Thus $q_{r}^{\prime}$ is given by

$$
q_{r}^{\prime}=-\frac{4 \sigma_{1}}{3 k_{1}} \frac{\partial T^{\prime 4}}{\partial y^{\prime}}
$$

where $k_{l}$ is the Rosseland mean absorption co-efficient and $\sigma_{l}$ is the Stefan-Boltzmann constant.

We assume that the temperature differences within the flow are sufficiently small. This results in the following approximations

$$
T^{\prime 4} \approx 4 T_{\infty}^{\prime 3} T^{\prime}-3 T_{\infty}^{\prime 4}
$$

From Eqs (2.5) and (2.6) we have

$$
\frac{\partial q_{r}^{\prime}}{\partial y^{\prime}}=-\frac{4 \sigma_{1}}{3 k_{1}} \frac{\partial^{2} T^{\prime 4}}{\partial y^{\prime 2}}=-\frac{16 \sigma_{1} T_{\infty}^{\prime 3}}{3 k_{1}} \frac{\partial^{2} T^{\prime}}{\partial y^{\prime 2}} .
$$

Thus the energy Eq.(2.2) reduces to

$$
\frac{\partial T^{\prime}}{\partial t^{\prime}}=\frac{k}{\rho C_{p}} \frac{\partial^{2} T^{\prime}}{\partial y^{\prime 2}}-\frac{Q_{0}}{\rho C_{p}}\left(T^{\prime}-T_{\infty}^{\prime}\right)+\frac{16 \sigma_{1} T_{\infty}^{\prime 3}}{3 k_{l} \rho C_{p}} \frac{\partial^{2} T^{\prime}}{\partial y^{\prime 2}} .
$$

Introducing the following non-dimensional quantities 


$$
\begin{aligned}
& y=\frac{y^{\prime}}{U_{0} t_{0}}, \quad u=\frac{u^{\prime}}{U_{0}}, \quad t=\frac{t^{\prime}}{t_{0}}, \quad \theta=\frac{T^{\prime}-T_{\infty}^{\prime}}{T_{w}^{\prime}-T_{\infty}^{\prime}}, \quad \phi=\frac{C^{\prime}-C_{\infty}^{\prime}}{C_{w}^{\prime}-C_{\infty}^{\prime}}, \\
& \mathrm{Gr}=\frac{g \beta v\left(T_{w}^{\prime}-T^{\prime}\right)}{U_{0}^{3}}, \quad \mathrm{Gm}=\frac{g \beta^{\prime} v\left(C_{w}^{\prime}-C_{\infty}^{\prime}\right)}{U_{0}^{3}}, \quad M=\frac{\sigma B_{0}^{2} \mathrm{v}}{\rho U_{0}^{2}}, \quad \operatorname{Pr}=\frac{\mu C p}{k}, \quad \mathrm{Sc}=\frac{v}{D}, \\
& Q=\frac{v Q_{0}}{\rho C p U_{0}^{2}}, \quad t_{0}=\frac{v}{U_{0}^{2}}, \quad K=\frac{K^{\prime} U_{0}^{2}}{v^{2}}, \quad N=\frac{k k_{1}}{4 \sigma_{1} T_{\infty}^{\prime 3}}, \quad \lambda=\frac{3 N+4}{3 N}, \quad K r=\frac{v K r^{\prime}}{U_{0}^{2}} .
\end{aligned}
$$

where $\mathrm{Gr}, \mathrm{Gm}, M, \operatorname{Pr}, \mathrm{Sc}, Q, t_{0}$ and $N$ are, respectively, the thermal Grashof number, the solutal Grashof number, the magnetic parameter, the Prandtl number, the Schmidt number, the heat absorption parameter, the characteristics time and radiation parameter.

Equations (2.1), (2.3) and (2.8) reduces to

$$
\begin{aligned}
& \frac{\partial u}{\partial t}=\frac{\partial^{2} u}{\partial y^{2}}+G r \theta+G m \phi-\left(M+\frac{1}{K}\right) u, \\
& \frac{\partial \theta}{\partial t}=\frac{\lambda}{\operatorname{Pr}} \frac{\partial^{2} \theta}{\partial y^{2}}-Q \theta \\
& \frac{\partial \phi}{\partial t}=\frac{1}{\operatorname{Sc}} \frac{\partial^{2} \phi}{\partial y^{2}}-K r \phi .
\end{aligned}
$$

The corresponding initial and boundary conditions in a non-dimensional form become

$$
\begin{aligned}
& u=0, \quad \theta=0, \quad \phi=0 \text { for } \quad y \geq 0 \text { and } t \leq 0, \\
& u=f(t), \quad \phi=1 \quad \text { at } y=0 \text { for } t>0, \\
& \theta=t \quad \text { at } y=0 \text { for } 0<t \leq 1, \\
& \theta=1 \quad \text { at } y=0 \text { for } t>1, \\
& u \rightarrow 0, \quad \theta \rightarrow 0, \quad \phi \rightarrow 0 \text { as } \quad y \rightarrow \infty \text { for } t>0 .
\end{aligned}
$$

The system of differential Eqs (2.9)-(2.11) together with the initial and boundary conditions (2.12a)(2.12e) describes our model for the MHD free convective heat and mass transfer flow of a viscous, incompressible, electrically conducting and heat absorbing fluid past a vertical plate through a porous medium with ramped wall temperature.

\section{Solution of the problem}

The set of Eqs (2.9)-(2.11) subject to the initial and boundary conditions (2.12a)-(2.12e) were solved analytically using the Laplace transforms. The exact solutions for species concentration $\phi(y, t)$, fluid temperature $\theta(y, t)$ and fluid velocity $u(y, t)$ are, respectively 


$$
\begin{aligned}
& \phi(y, t)=\frac{1}{2}\left[e^{y \sqrt{\mathrm{ScKr}}} \operatorname{erfc}\left(\frac{y}{2} \sqrt{\frac{\mathrm{Sc}}{t}}+\sqrt{\mathrm{Kr} t}\right)+e^{-y \sqrt{\mathrm{ScKr}}} \operatorname{erfc}\left(\frac{y}{2} \sqrt{\frac{\mathrm{Sc}}{t}}-\sqrt{\mathrm{Kr} t}\right)\right], \\
& \theta(y, t)=\theta_{1}(y, t)-H(t-l) \theta_{l}(y, t-l) \\
& u(y, t)=P(y, t)+\alpha_{1}\left[P_{l}(y, t)-H(t-l) P_{l}(y, t-l)\right]+\alpha_{2} P_{2}(y, t)
\end{aligned}
$$

where

$$
\begin{aligned}
& P(y, t)=L^{-1}\left\{\bar{f}(s) e^{-y \sqrt{s+A_{l}}}\right\} \\
& \theta_{l}(y, t)=\frac{1}{2}\left[\left(t+\frac{y}{2} \sqrt{\frac{\operatorname{Pr}}{\lambda Q}}\right) e^{y \sqrt{\frac{\operatorname{Pr} Q}{\lambda}}} \operatorname{erfc}\left(\frac{y}{2} \sqrt{\frac{\operatorname{Pr}}{\lambda t}}+\sqrt{Q t}\right)+\right. \\
& \left.+\left(t-\frac{y}{2} \sqrt{\frac{\operatorname{Pr}}{\lambda Q}}\right) e^{-y \sqrt{\frac{\operatorname{Pr} Q}{\lambda}}} \operatorname{erfc}\left(\frac{y}{2} \sqrt{\frac{\operatorname{Pr}}{\lambda t}}-\sqrt{Q t}\right)\right] .
\end{aligned}
$$

Here, $H(t-1), \operatorname{erfc}(x)$ and $L^{-1}$ are the Heaviside unit step function, the complimentary error function and the inverse Laplace transform operator, respectively. The other terms are calculated but not presented here for the sake of brevity.

\subsection{Solution in the case of isothermal plate}

Equations (3.1)-(3.3) represent the analytical solutions for species concentration, fluid temperature and fluid velocity for free convection heat and mass transfer flow of a viscous, incompressible, electrically conducting and heat absorbing fluid past a flat plate through a porous medium with ramped temperature taking chemical reaction and thermal radiation into account. In order to highlight the effects of the ramped temperature distribution on the fluid flow, it is justified to compare such a flow with the flow near a moving plate with uniform temperature. Keeping in view the assumptions made in this paper, the solution for species concentration is given by Eq.(3.1), however, the fluid temperature and fluid velocity for free convection near an isothermal plate is obtained and expressed in the following form

$$
\begin{aligned}
& \theta(y, t)=\frac{1}{2}\left[e^{y \sqrt{\frac{\operatorname{Pr} Q}{\lambda}}} \operatorname{erfc}\left(\frac{y}{2} \sqrt{\frac{\operatorname{Pr}}{\lambda t}}+\sqrt{Q t}\right)+e^{\left.-y \sqrt{\frac{\operatorname{Pr} Q}{\lambda}} \operatorname{erfc}\left(\frac{y}{2} \sqrt{\frac{\operatorname{Pr}}{\lambda t}}-\sqrt{Q t}\right)\right],}\right. \\
& u(y, t)=P(y, t)+\alpha_{1} P_{3}(y, t)+\alpha_{2} P_{2}(y, t) .
\end{aligned}
$$

\subsection{Sherwood number}

The Sherwood number Sh, which measures the rate of mass transfer at the plate, is given by

$$
\mathrm{Sh}=-\left(\frac{\partial \phi}{\partial y}\right)_{y=0}=\sqrt{\mathrm{ScKr}} \operatorname{erf}(\sqrt{\mathrm{Kr} t})+\sqrt{\frac{\mathrm{Sc}}{\pi t}} e^{-\mathrm{Kr} t}
$$




\subsection{Nusselt number}

The Nusselt number $\mathrm{Nu}$ measures the rate of heat transfer at the plate and for a ramped temperature plate is given by

$$
\mathrm{Nu}=-\left(\frac{\partial \theta}{\partial y}\right)_{y=0}=-\left[\theta_{2}(0, t)-H(t-1) \theta_{2}(0, t-1)\right]
$$

where, $\quad \theta_{2}(0, t)=-\left[t \sqrt{\frac{\operatorname{Pr} Q}{\lambda}} \operatorname{erf}(\sqrt{Q t})+\frac{1}{2} \sqrt{\frac{\operatorname{Pr}}{\lambda Q}} \operatorname{erf}(\sqrt{Q t})+t \sqrt{\frac{\operatorname{Pr}}{\pi \lambda t}} e^{-Q t}\right]$.

In the case of an isothermal plate, the Nusselt number is given by

$$
\mathrm{Nu}_{i}=\sqrt{\frac{\operatorname{Pr} Q}{\lambda}} \operatorname{erf}(\sqrt{Q t})+\sqrt{\frac{\operatorname{Pr}}{\pi \lambda t}} e^{-Q t} .
$$

Equations (3.1)-(3.5) represent the analytical solutions for the flow due to time-dependent movement of the vertical flat plate through a porous medium. In order to get some physical understanding of the flow behaviour, some particular cases of time-dependent movements of the plate are discussed below.

\subsection{Plate moving with uniform velocity}

Let us consider the plate moving with a uniform velocity $f(t)=H(t)$. The fluid velocity of the flow for the ramped temperature plate is obtained as

$$
u(y, t)=P_{4}(y, t)+\alpha_{1}\left[P_{l}(y, t)-H(t-1) P_{1}(y, t-1)\right]+\alpha_{2} P_{2}(y, t),
$$

and for the isothermal plate the fluid velocity is

$$
u(y, t)=P_{4}(y, t)+\alpha_{1} P_{3}(y, t)+\alpha_{2} P_{2}(y, t) .
$$

The expression for the skin friction for the ramped temperature plate is given by

$$
\tau_{l}=\left(\frac{\partial u}{\partial y}\right)_{y=0}=Q_{1}(t)+\alpha_{1}\left[F_{l}(t)-H(t-1) F_{1}(t-1)\right]+\alpha_{2} F_{2}(t),
$$

and for the isothermal plate it is given by

$$
\tau_{l i}=Q_{1}(t)+\alpha_{1} F_{3}(t)+\alpha_{2} F_{2}(t) .
$$




\subsection{Plate moving with uniform acceleration}

Let us consider the plate moving with uniform acceleration $f(t)=t H(t)$. The fluid velocity of the flow for the ramped temperature plate is obtained as

$$
u(y, t)=P_{5}(y, t)+\alpha_{1}\left[P_{l}(y, t)-H(t-1) P_{1}(y, t-1)\right]+\alpha_{2} P_{2}(y, t),
$$

and for the isothermal plate the fluid velocity it is

$$
u(y, t)=P_{5}(y, t)+\alpha_{1} P_{3}(y, t)+\alpha_{2} P_{2}(y, t) .
$$

The expression for the skin friction for the ramped temperature plate is given by

$$
\tau_{2}=\left(\frac{\partial u}{\partial y}\right)_{y=0}=Q_{2}(t)+\alpha_{1}\left[F_{l}(t)-H(t-1) F_{1}(t-1)\right]+\alpha_{2} F_{2}(t),
$$

and for the isothermal plate it is given by

$$
\tau_{2 i}=Q_{2}(t)+\alpha_{1} F_{3}(t)+\alpha_{2} F_{2}(t) .
$$

\subsection{Plate moving with periodic acceleration}

Let us consider the plate moving with periodic acceleration $f(t)=\cos \omega t H(t)$. The fluid velocity of the flow for the ramped temperature plate is obtained as

$$
u(y, t)=P_{6}(y, t)+\alpha_{1}\left[P_{l}(y, t)-H(t-1) P_{l}(y, t-1)\right]+\alpha_{2} P_{2}(y, t),
$$

and for the isothermal plate the fluid velocity is

$$
u(y, t)=P_{6}(y, t)+\alpha_{1} P_{3}(y, t)+\alpha_{2} P_{2}(y, t) .
$$

The expression for the skin friction for the ramped temperature plate is given by

$$
\tau_{3}=\left(\frac{\partial u}{\partial y}\right)_{y=0}=Q_{3}(t)+\alpha_{1}\left[F_{1}(t)-H(t-1) F_{1}(t-1)\right]+\alpha_{2} F_{2}(t),
$$

and for the isothermal plate it is given by

$$
\tau_{3 i}=Q_{3}(t)+\alpha_{1} F_{3}(t)+\alpha_{2} F_{2}(t) .
$$

\section{Results and discussion}

In order to get the physical understanding of the problem and for the purpose of discussing the results, numerical calculations have been carried out for the non-dimensional velocity $(u)$, the temperature $(\theta)$ and the species concentration $(\phi)$. They are depicted in Figs 2-12 for various values of 
the thermal Grashof number (Gr), the solutal Grashof number $(\mathrm{Gm})$, the Prandtl number $(\mathrm{Pr})$, the heat absorption co-efficient $(Q)$, the Hartmann number $(\mathrm{M})$, the chemical reaction parameter $(\mathrm{Kr})$, the radiation parameter $(N)$ and $\omega=\pi / 2$ for both ramped temperature and isothermal plates. In order to verify the validity and accuracy of the present analysis, results for the skin friction $\tau$ were compared with those reported by Nandkeolyar et al. [29] in all three cases. The values of the skin-friction for the plate moving with uniform velocity, plate moving with uniform acceleration and plate moving with periodic acceleration are depicted in Tabs 1-3, respectively for both ramped temperature and isothermal plates. The values of the Nusselt number and the Sherwood number are depicted in Tab.4 for both ramped temperature and isothermal plates.

Table1. Skin friction when $f(t)=H(t)$ for $\mathrm{Sc}=0.6$.

\begin{tabular}{ccccccccccc}
\hline$t$ & $\mathrm{Gr}$ & $\mathrm{Gm}$ & $\operatorname{Pr}$ & $Q$ & $M$ & $K$ & $K r$ & $N$ & $\tau_{1}$ & $\tau_{1 i}$ \\
\hline $\boldsymbol{0 . 3}$ & 2 & 2 & 0.71 & 1 & 3 & 0.2 & 1 & 1 & -2.1046 & -1.5826 \\
$\mathbf{0 . 5}$ & 2 & 2 & 0.71 & 1 & 3 & 0.2 & 1 & 1 & -1.9883 & -1.5781 \\
$\mathbf{0 . 7}$ & 2 & 2 & 0.71 & 1 & 3 & 0.2 & 1 & 1 & -1.8855 & -1.5695 \\
0.7 & $\mathbf{2}$ & 2 & 0.71 & 1 & 3 & 0.2 & 1 & 1 & -1.8855 & -1.5695 \\
0.7 & $\mathbf{4}$ & 2 & 0.71 & 1 & 3 & 0.2 & 1 & 1 & -1.5465 & -0.9745 \\
0.7 & $\mathbf{6}$ & 2 & 0.71 & 1 & 3 & 0.2 & 1 & 1 & -1.2074 & -0.3495 \\
0.7 & 2 & $\mathbf{2}$ & 0.71 & 1 & 3 & 0.2 & 1 & 1 & -1.8855 & -1.5695 \\
0.7 & 2 & $\mathbf{4}$ & 0.71 & 1 & 3 & 0.2 & 1 & 1 & -1.2815 & -0.9955 \\
0.7 & 2 & $\mathbf{6}$ & 0.71 & 1 & 3 & 0.2 & 1 & 1 & -0.6775 & -0.3915 \\
0.7 & 2 & 2 & $\mathbf{0 . 5}$ & 1 & 3 & 0.2 & 1 & 1 & -1.8715 & -1.5894 \\
0.7 & 2 & 2 & $\mathbf{0 . 7 1}$ & 1 & 3 & 0.2 & 1 & 1 & -1.8855 & -1.5695 \\
0.7 & 2 & 2 & 7 & 1 & 3 & 0.2 & 1 & 1 & -1.998 & -1.8223 \\
0.7 & 2 & 2 & 0.71 & $\mathbf{1}$ & 3 & 0.2 & 1 & 1 & -1.8934 & -1.6074 \\
0.7 & 2 & 2 & 0.71 & $\mathbf{3}$ & 3 & 0.2 & 1 & 1 & -1.91 & -1.6937 \\
0.7 & 2 & 2 & 0.71 & $\mathbf{5}$ & 3 & 0.2 & 1 & 1 & -1.9244 & -1.7377 \\
0.7 & 2 & 2 & 0.71 & 1 & $\mathbf{3}$ & 0.2 & 1 & 1 & -1.8855 & -1.5695 \\
0.7 & 2 & 2 & 0.71 & 1 & $\mathbf{5}$ & 0.2 & 1 & 1 & -2.2976 & -2.0501 \\
0.7 & 2 & 2 & 0.71 & 1 & 7 & 0.2 & 1 & 1 & -2.6602 & -2.4398 \\
0.7 & 2 & 2 & 0.71 & 1 & 3 & $\mathbf{0 . 2}$ & 1 & 1 & -1.8855 & -1.5695 \\
0.7 & 2 & 2 & 0.71 & 1 & 3 & $\mathbf{1}$ & 1 & 1 & -0.8057 & -0.3625 \\
0.7 & 2 & 2 & 0.71 & 1 & 3 & $\mathbf{2}$ & 1 & 1 & -0.6329 & -0.1551 \\
0.7 & 2 & 2 & 0.71 & 1 & 3 & 0.2 & $\mathbf{1}$ & 1 & -1.8855 & -1.5695 \\
0.7 & 2 & 2 & 0.71 & 1 & 3 & 0.2 & $\mathbf{3}$ & 1 & -1.9932 & -1.7072 \\
0.7 & 2 & 2 & 0.71 & 1 & 3 & 0.2 & $\mathbf{5}$ & 1 & -2.0463 & -1.7603 \\
0.7 & 2 & 2 & 0.71 & 1 & 3 & 0.2 & 1 & $\mathbf{1}$ & -1.8855 & -1.5695 \\
0.7 & 2 & 2 & 0.71 & 1 & 3 & 0.2 & 1 & $\mathbf{3}$ & -1.9063 & -1.6143 \\
0.7 & 2 & 2 & 0.71 & 1 & 3 & 0.2 & 1 & $\mathbf{5}$ & -1.9121 & -1.6185 \\
\hline & & & & & & & & & &
\end{tabular}


Table2. Skin friction when $f(t)=t H(t)$ for $\mathrm{Sc}=0.6$.

\begin{tabular}{ccccccccccc}
\hline$t$ & $\mathrm{Gr}$ & $\mathrm{Gm}$ & $\operatorname{Pr}$ & $Q$ & $M$ & $K$ & $K r$ & $N$ & $\tau_{2}$ & $\tau_{2 i}$ \\
\hline $\mathbf{0 . 3}$ & 2 & 2 & 0.71 & 1 & 3 & 0.2 & 1 & 1 & -0.2873 & 0.2347 \\
$\mathbf{0 . 5}$ & 2 & 2 & 0.71 & 1 & 3 & 0.2 & 1 & 1 & -0.7493 & -0.3392 \\
$\mathbf{0 . 7}$ & 2 & 2 & 0.71 & 1 & 3 & 0.2 & 1 & 1 & -1.2136 & -0.9276 \\
0.7 & $\mathbf{2}$ & 2 & 0.71 & 1 & 3 & 0.2 & 1 & 1 & -1.2136 & -0.9276 \\
0.7 & $\mathbf{4}$ & 2 & 0.71 & 1 & 3 & 0.2 & 1 & 1 & -0.8745 & -0.3025 \\
0.7 & $\mathbf{6}$ & 2 & 0.71 & 1 & 3 & 0.2 & 1 & 1 & -0.5354 & 0.3225 \\
0.7 & 2 & $\mathbf{2}$ & 0.71 & 1 & 3 & 0.2 & 1 & 1 & -1.2136 & -0.9276 \\
0.7 & 2 & $\mathbf{4}$ & 0.71 & 1 & 3 & 0.2 & 1 & 1 & -0.6095 & -0.3236 \\
0.7 & 2 & $\mathbf{6}$ & 0.71 & 1 & 3 & 0.2 & 1 & 1 & -0.0055 & 0.2805 \\
0.7 & 2 & 2 & $\mathbf{0 . 5}$ & 1 & 3 & 0.2 & 1 & 1 & -1.1995 & -0.9174 \\
0.7 & 2 & 2 & $\mathbf{0 . 7 1}$ & 1 & 3 & 0.2 & 1 & 1 & -1.2136 & -0.9276 \\
0.7 & 2 & 2 & 7 & 1 & 3 & 0.2 & 1 & 1 & -1.326 & -1.1504 \\
0.7 & 2 & 2 & 0.71 & $\mathbf{1}$ & 3 & 0.2 & 1 & 1 & -1.2136 & -0.9276 \\
0.7 & 2 & 2 & 0.71 & $\mathbf{3}$ & 3 & 0.2 & 1 & 1 & -1.2301 & -1.0138 \\
0.7 & 2 & 2 & 0.71 & $\mathbf{5}$ & 3 & 0.2 & 1 & 1 & -1.2446 & -1.0579 \\
0.7 & 2 & 2 & 0.71 & 1 & $\mathbf{3}$ & 0.2 & 1 & 1 & -1.2136 & -0.9276 \\
0.7 & 2 & 2 & 0.71 & 1 & $\mathbf{5}$ & 0.2 & 1 & 1 & -1.507 & -1.2595 \\
0.7 & 2 & 2 & 0.71 & 1 & 7 & 0.2 & 1 & 1 & -1.7653 & -1.5449 \\
0.7 & 2 & 2 & 0.71 & 1 & 3 & $\mathbf{0 . 2}$ & 1 & 1 & -1.2136 & -0.9276 \\
0.7 & 2 & 2 & 0.71 & 1 & 3 & $\mathbf{1}$ & 1 & 1 & -0.4497 & -0.0065 \\
0.7 & 2 & 2 & 0.71 & 1 & 3 & $\mathbf{2}$ & 1 & 1 & -0.3294 & 0.1485 \\
0.7 & 2 & 2 & 0.71 & 1 & 3 & 0.2 & $\mathbf{1}$ & 1 & -1.2136 & -0.9276 \\
0.7 & 2 & 2 & 0.71 & 1 & 3 & 0.2 & $\mathbf{3}$ & 1 & -1.3212 & -1.0353 \\
0.7 & 2 & 2 & 0.71 & 1 & 3 & 0.2 & $\mathbf{5}$ & 1 & -1.374 & -1.0883 \\
0.7 & 2 & 2 & 0.71 & 1 & 3 & 0.2 & 1 & $\mathbf{1}$ & -1.2136 & -0.9276 \\
0.7 & 2 & 2 & 0.71 & 1 & 3 & 0.2 & 1 & $\mathbf{3}$ & -1.2343 & -0.9424 \\
0.7 & 2 & 2 & 0.71 & 1 & 3 & 0.2 & 1 & $\mathbf{5}$ & -1.2402 & -0.9465 \\
\hline & & & & & & & & & &
\end{tabular}


Table 3. Skin friction when $f(t)=\cos \omega t H(t)$ for $\mathrm{Sc}=0.6$.

\begin{tabular}{ccccccccccc}
\hline$t$ & $\mathrm{Gr}$ & $\mathrm{Gm}$ & $\operatorname{Pr}$ & $Q$ & $M$ & $K$ & $K r$ & $N$ & $\tau_{3}$ & $\tau_{3 i}$ \\
\hline $\mathbf{0 . 3}$ & 2 & 2 & 0.71 & 1 & 3 & 0.2 & 1 & 1 & -0.2873 & 0.2347 \\
$\mathbf{0 . 5}$ & 2 & 2 & 0.71 & 1 & 3 & 0.2 & 1 & 1 & -0.7493 & -0.3392 \\
$\mathbf{0 . 7}$ & 2 & 2 & 0.71 & 1 & 3 & 0.2 & 1 & 1 & -1.2136 & -0.9276 \\
0.7 & $\mathbf{2}$ & 2 & 0.71 & 1 & 3 & 0.2 & 1 & 1 & -1.2136 & -0.9276 \\
0.7 & $\mathbf{4}$ & 2 & 0.71 & 1 & 3 & 0.2 & 1 & 1 & -0.8745 & -0.3025 \\
0.7 & $\mathbf{6}$ & 2 & 0.71 & 1 & 3 & 0.2 & 1 & 1 & -0.5354 & 0.3225 \\
0.7 & 2 & $\mathbf{2}$ & 0.71 & 1 & 3 & 0.2 & 1 & 1 & -1.2136 & -0.9276 \\
0.7 & 2 & $\mathbf{4}$ & 0.71 & 1 & 3 & 0.2 & 1 & 1 & -0.6095 & -0.3236 \\
0.7 & 2 & $\mathbf{6}$ & 0.71 & 1 & 3 & 0.2 & 1 & 1 & -0.0055 & 0.2805 \\
0.7 & 2 & 2 & $\mathbf{0 . 5}$ & 1 & 3 & 0.2 & 1 & 1 & -1.1995 & -0.9174 \\
0.7 & 2 & 2 & $\mathbf{0 . 7 1}$ & 1 & 3 & 0.2 & 1 & 1 & -1.2136 & -0.9276 \\
0.7 & 2 & 2 & 7 & 1 & 3 & 0.2 & 1 & 1 & -1.326 & -1.1504 \\
0.7 & 2 & 2 & 0.71 & $\mathbf{1}$ & 3 & 0.2 & 1 & 1 & -1.2136 & -0.9276 \\
0.7 & 2 & 2 & 0.71 & $\mathbf{3}$ & 3 & 0.2 & 1 & 1 & -1.2301 & -1.0138 \\
0.7 & 2 & 2 & 0.71 & $\mathbf{5}$ & 3 & 0.2 & 1 & 1 & -1.2446 & -1.0579 \\
0.7 & 2 & 2 & 0.71 & 1 & $\mathbf{3}$ & 0.2 & 1 & 1 & -1.2136 & -0.9276 \\
0.7 & 2 & 2 & 0.71 & 1 & $\mathbf{5}$ & 0.2 & 1 & 1 & -1.507 & -1.2595 \\
0.7 & 2 & 2 & 0.71 & 1 & 7 & 0.2 & 1 & 1 & -1.7653 & -1.5449 \\
0.7 & 2 & 2 & 0.71 & 1 & 3 & $\mathbf{0 . 2}$ & 1 & 1 & -1.2136 & -0.9276 \\
0.7 & 2 & 2 & 0.71 & 1 & 3 & $\mathbf{1}$ & 1 & 1 & -0.4497 & -0.0065 \\
0.7 & 2 & 2 & 0.71 & 1 & 3 & $\mathbf{2}$ & 1 & 1 & -0.3294 & 0.1485 \\
0.7 & 2 & 2 & 0.71 & 1 & 3 & 0.2 & $\mathbf{1}$ & 1 & -1.2136 & -0.9276 \\
0.7 & 2 & 2 & 0.71 & 1 & 3 & 0.2 & $\mathbf{3}$ & 1 & -1.3212 & -1.0353 \\
0.7 & 2 & 2 & 0.71 & 1 & 3 & 0.2 & $\mathbf{5}$ & 1 & -1.3743 & -1.0883 \\
0.7 & 2 & 2 & 0.71 & 1 & 3 & 0.2 & 1 & $\mathbf{1}$ & -1.2136 & -0.9276 \\
0.7 & 2 & 2 & 0.71 & 1 & 3 & 0.2 & 1 & $\mathbf{3}$ & -1.2343 & -0.9424 \\
0.7 & 2 & 2 & 0.71 & 1 & 3 & 0.2 & 1 & $\mathbf{5}$ & -1.2402 & -0.9465 \\
\hline & & & & & & & & & &
\end{tabular}

Table 4. The Nusselt number and Sherwood number.

\begin{tabular}{ccccccccc}
\hline$T$ & $\operatorname{Pr}$ & $Q$ & $N$ & $\mathrm{Sc}$ & $K r$ & $\mathrm{Nu}$ & $\mathrm{Nu}_{i}$ & $\mathrm{Sh}$ \\
\hline $\mathbf{0 . 3}$ & 0.71 & 1 & 1 & 0.6 & 1 & 0.374 & 0.7306 & 1.026 \\
$\mathbf{0 . 5}$ & 0.71 & 1 & 1 & 0.6 & 1 & 0.5101 & 0.6435 & 0.9037 \\
$\mathbf{0 . 7}$ & 0.71 & 1 & 1 & 0.6 & 1 & 0.6346 & 0.6058 & 0.8506 \\
0.7 & $\mathbf{0 . 5}$ & 1 & 1 & - & - & 0.5325 & 0.5083 & - \\
0.7 & $\mathbf{0 . 7 1}$ & 1 & 1 & - & - & 0.6346 & 0.6058 & - \\
0.7 & 7 & 1 & 1 & - & - & 1.9924 & 1.902 & - \\
0.7 & 0.71 & $\mathbf{1}$ & 1 & - & - & 0.6346 & 0.6058 & - \\
0.7 & 0.71 & $\mathbf{3}$ & 1 & - & - & 0.8265 & 0.9624 & - \\
0.7 & 0.71 & $\mathbf{5}$ & 1 & - & - & 0.9866 & 1.2346 & - \\
0.7 & 0.71 & 1 & $\mathbf{1}$ & - & - & 0.6346 & 0.6058 & - \\
0.7 & 0.71 & 1 & $\mathbf{3}$ & - & - & 0.8065 & 0.7699 & - \\
0.7 & 0.71 & 1 & $\mathbf{5}$ & - & - & 0.8612 & 0.8222 & - \\
0.7 & - & - & - & $\mathbf{0 . 4}$ & 1 & - & - & 0.6945 \\
0.7 & - & - & - & $\mathbf{0 . 6}$ & 1 & - & - & 0.8506 \\
0.7 & - & - & - & $\mathbf{0 . 8}$ & 1 & - & - & 0.9699 \\
0.7 & - & - & - & 0.6 & $\mathbf{1}$ & - & - & 0.8506 \\
0.7 & - & - & - & 0.6 & $\mathbf{3}$ & - & - & 1.3514 \\
0.7 & - & - & - & 0.6 & $\mathbf{5}$ & - & - & 1.7337 \\
\hline
\end{tabular}


Figure 2 shows that for both ramped temperature and isothermal plates, the fluid velocity $u(y, t)$ increases with an increase in time $t$ for all cases except in the case of periodic acceleration of the plate. In this case, the fluid velocity near the plate decreases with an increase in time $t$. Thus, in general, it can be calculated that the fluid velocity increases in all three cases with time $t$. Figures 3-4 show the effect of the thermal Grashof number Gr and the solutal Grashof number Gm, respectively, for both ramped temperature and isothermal plates. In all three cases, the fluid velocity increases with an increase in $\mathrm{Gr}$ and $\mathrm{Gm}$. Gr represents the relative strength of the thermal buoyancy force to the viscous force and $\mathrm{Gm}$ represents the relative strength of the concentration buoyancy force to the viscous force. Therefore, $\mathrm{Gr}$ increases on increasing the strengths of the thermal buoyancy force and Gm increases on increasing the strength of the concentration buoyancy force. In this problem, natural convection flow is induced by thermal and concentration buoyancy forces, therefore the thermal and concentration buoyancy forces tend to accelerate the fluid velocity throughout the boundary layer region which is clearly evident from Figs 3-4. Figures 5-7 demonstrate the effect of the Prandtl number (Pr), $Q$ and $M$ on fluid velocity, respectively, in all three cases. It is perceived from Fig. 5 that fluid velocity decreases on increasing $\operatorname{Pr}$ throughout the boundary layer region. Physically, this is possible because fluids with a high Prandtl number have greater viscosity, which makes the fluid thick and hence move slowly. From Fig. 6 we observe that the velocity decreases with an increase in the heat absorption parameter $Q$, because, in the presence of the heat absorption, the buoyancy force decreases the velocity profiles. Physically, the presence of heat absorption coefficient has the tendency to reduce the fluid velocity. This cause the thermal buoyancy effects to decrease resulting in a net reduction in the fluid velocity. From Fig. 7 it is observed that an increase in the value of $M$ results in a decrease in the velocity profiles. This is due to the application of a magnetic field to an electrically conducting fluid which produces a dragline force that causes a reduction in the fluid velocity. The effects of chemical reaction $K r$ and thermal radiation $\mathrm{N}$ are shown in Figs 8-9, respectively, for both ramped temperature and isothermal plates. From Fig.8 it is quite clear that increasing the chemical reaction parameter tends to decrease the velocity of the fluid. This means that the chemical reaction decelerates the fluid motion. Consequently, less flow is induced along the plate resulting in a decrease in the fluid velocity in the boundary layer. It should be mentioned here that physically positive values of $K r$ imply a destructive reaction and negative values of $K r$ imply a generative reaction. We studied the case of a destructive chemical reaction $(\mathrm{Kr})$. From Fig.9 it is observed that the fluid velocity decreases with an increase in the thermal radiation parameter. This is in agreement with the fact that the thermal boundary layer thickness decreases with an increase in the radiation parameter. An increase in the permeability parameter $K$ leads to an increase in the velocity in the boundary layer region for both ramped temperature and isothermal plates as shown in Fig.10. This is due to fact that an increase in $K$ implies that there is a decrease in the resistance of the porous medium which tends to accelerate the velocity in the boundary layer region for both ramped temperature and isothermal plates. We further note from Figs 2-10 that the fluid velocity is higher in the case of a flow past an isothermal plate in comparison to a flow past a ramped temperature plate. The effects of the magnetic field, thermal Grashof number, solutal Grashof number, heat absorption parameter, the Prandtl number and time on the fluid velocity in the case of a flow past a flat plate are in agreement with those of Nandkeolyar et al. [29]. 


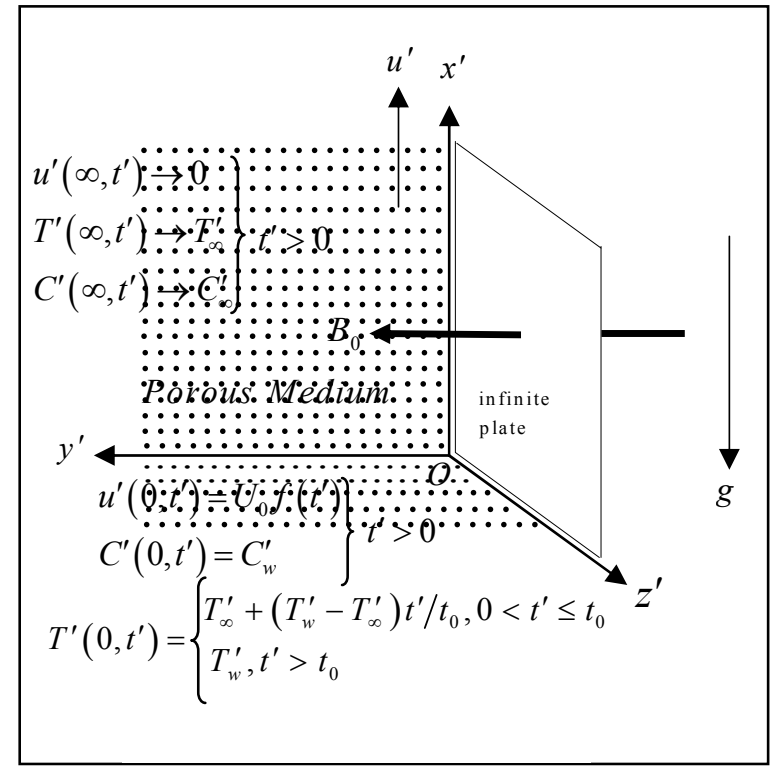

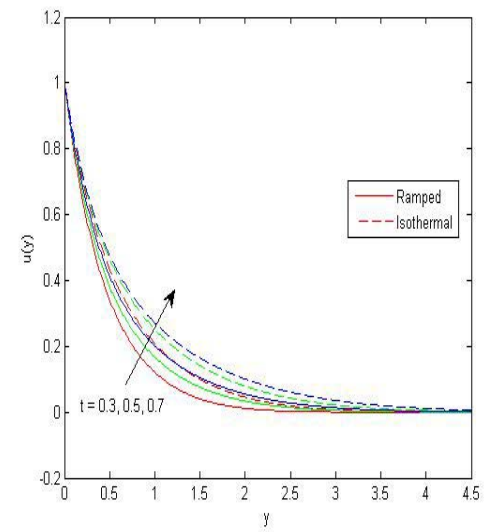

(a)

Fig.1 Geometry of the problem.

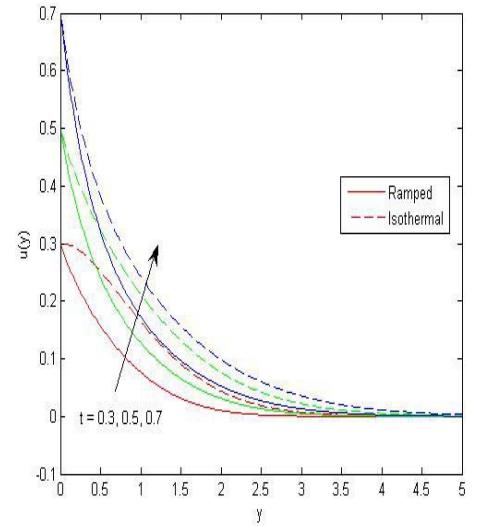

(b)

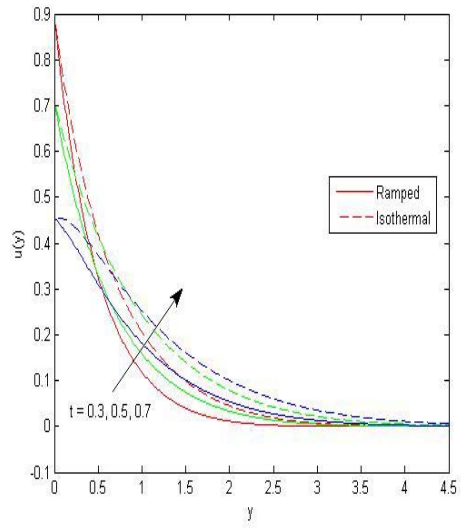

(c)

Fig.2. Effect of variation in time $t$ on the fluid velocity $u(y, t)$ when (a) $f(t)=H(t)$, (b) $f(t)=t H(t)$ and (c) $f(t)=\cos \omega t H(t)$ for $\mathrm{Sc}=0.6, Q=1, \operatorname{Pr}=0.71, \mathrm{Gr}=2, \mathrm{Gm}=2, K=0.2, K r=1, N=1, M=3$.

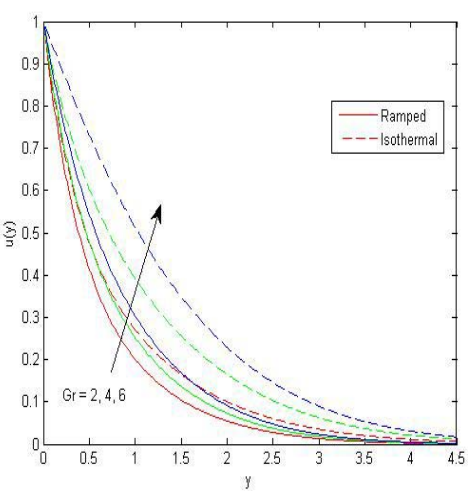

(a)

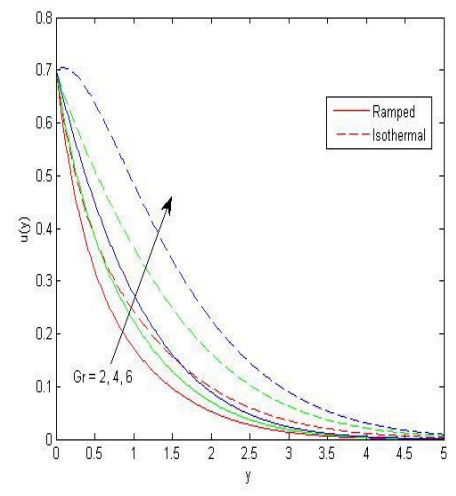

(b)

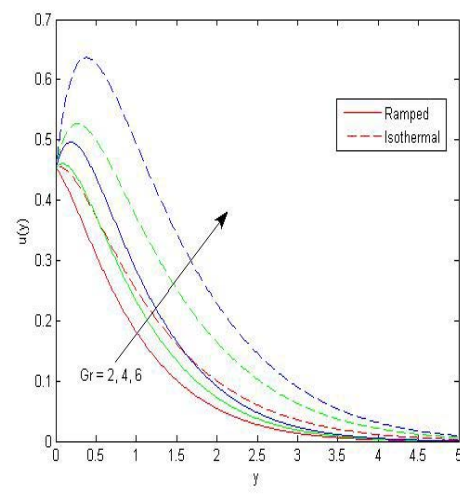

(c)

Fig.3. Effect of variation of the Grashof number for heat transfer Gr on the fluid velocity $u(y, t)$ when (a) $f(t)=H(t)$, (b) $f(t)=t H(t)$ and (c) $f(t)=\cos \omega t H(t)$ for $\mathrm{Sc}=0.6, Q=1, \operatorname{Pr}=0.71, G m=2, K=0.2, K r=1, N=1, M=3, t=0.7$. 


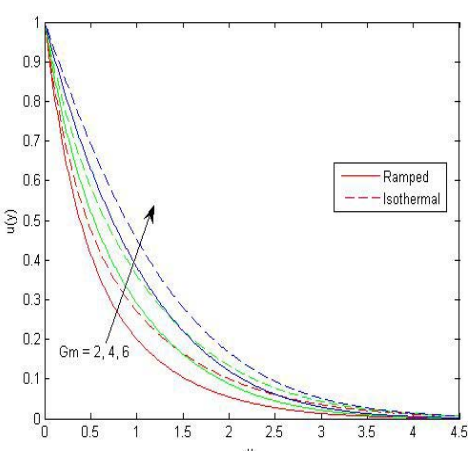

(a)

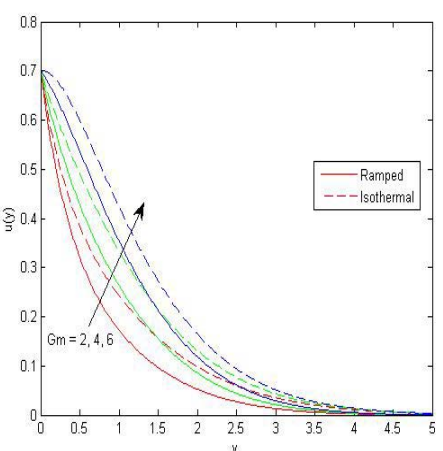

(b)

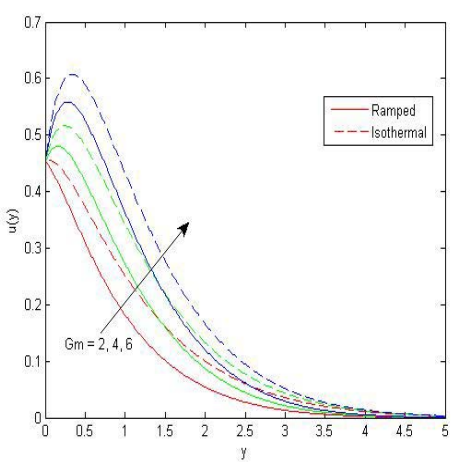

(c)

Fig.4. Effect of variation of the Grashof number for mass transfer Gm on the fluid velocity $u(y, t)$ when (a) $f(t)=H(t)$, (b) $f(t)=t H(t)$ and (c) $f(t)=\cos \omega t H(t)$ for $\mathrm{Sc}=0.6, Q=1, \operatorname{Pr}=0.71, \mathrm{Gr}=2, K=0.2, K r=1$, $N=1, M=3, t=0.7$.

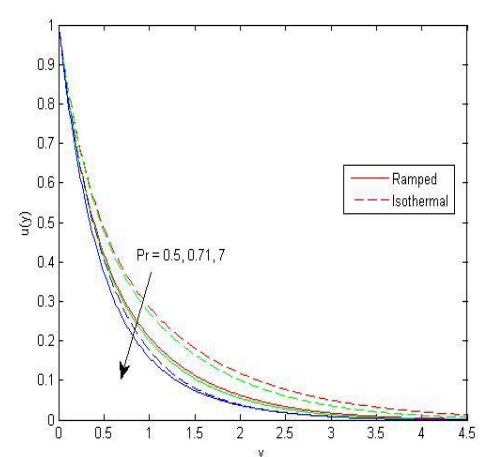

(a)

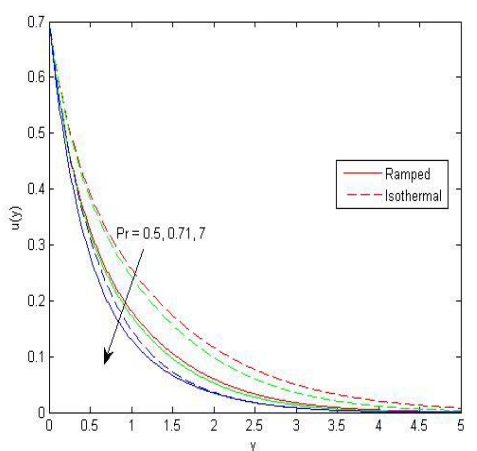

(b)

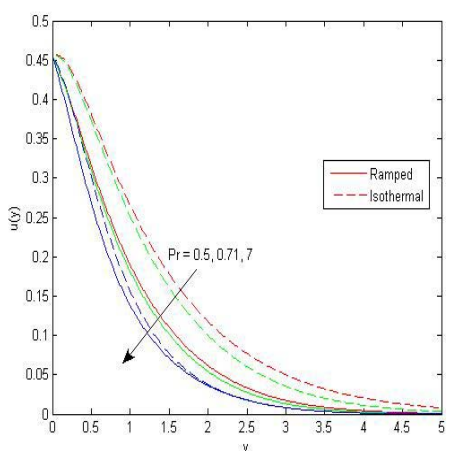

(c)

Fig.5. Effect of variation of the Prandtl number $\operatorname{Pr}$ on the fluid velocity $u(y, t)$ when (a) $f(t)=H(t)$, (b) $f(t)=t H(t)$ and (c) $f(t)=\cos \omega t H(t)$ for $\mathrm{Sc}=0.6, Q=1, \mathrm{Gr}=2, G m=2, K=0.2, K r=1, N=1, M=3$, $t=0.7$.

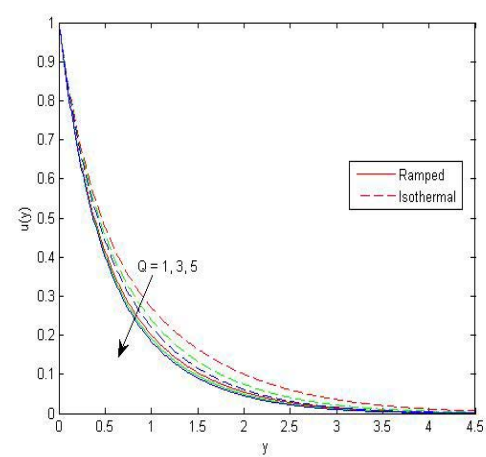

(a)

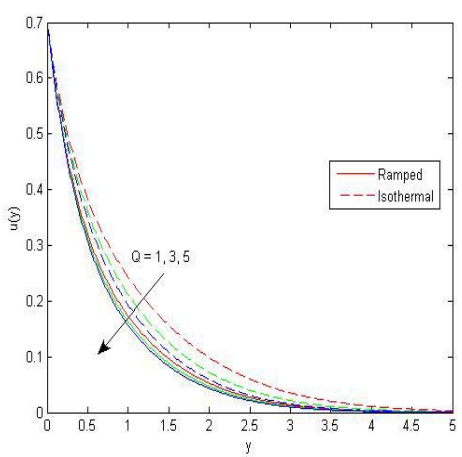

(b)

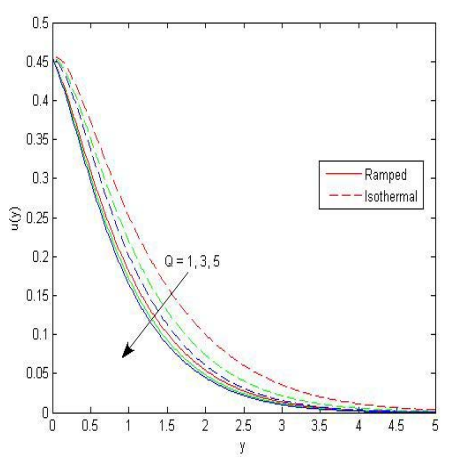

(c)

Fig.6. Effect of variation in the heat absorption parameter $Q$ on the fluid velocity $u(y, t)$ when (a) $f(t)=H(t)$, (b) $f(t)=t H(t)$ and (c) $f(t)=\cos \omega t H(t)$ for $\mathrm{Sc}=0.6, \mathrm{Pr}=0.71, \mathrm{Gr}=2, G m=2, K=0.2, K r=1, N=1, M=3$, $t=0.7$. 


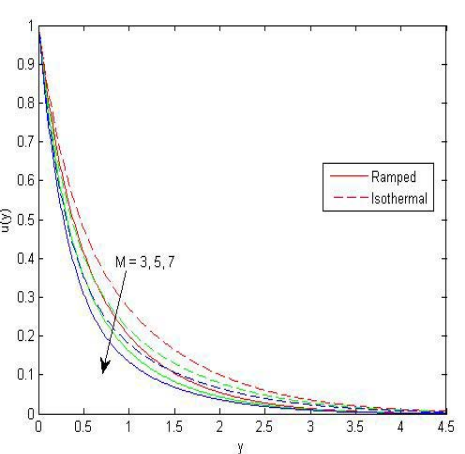

(a)

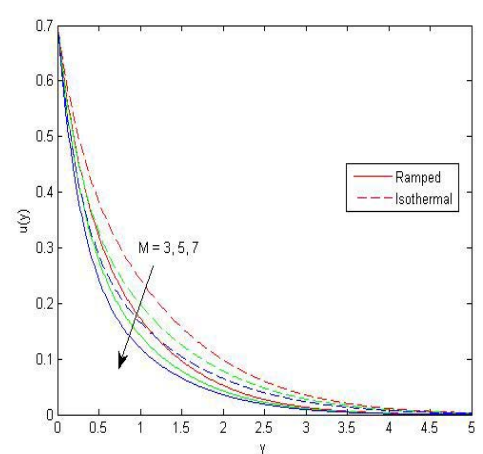

(b)

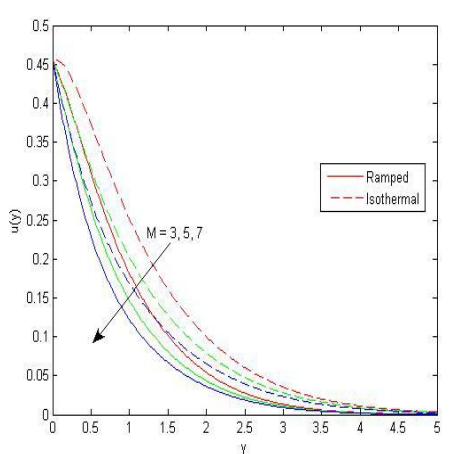

(c)

Fig.7. Effect of variation in the Hartmann number $\mathrm{M}$ on the fluid velocity $u(y, t)$ when (a) $f(t)=H(t)$, (b) $f(t)=t H(t)$ and (c) $f(t)=\cos \omega t H(t)$ for $\mathrm{Sc}=0.6, Q=1, \mathrm{Pr}=0.71, \mathrm{Gr}=2, G m=2, K=0.2, K r=1, N=1$, $t=0.7$.

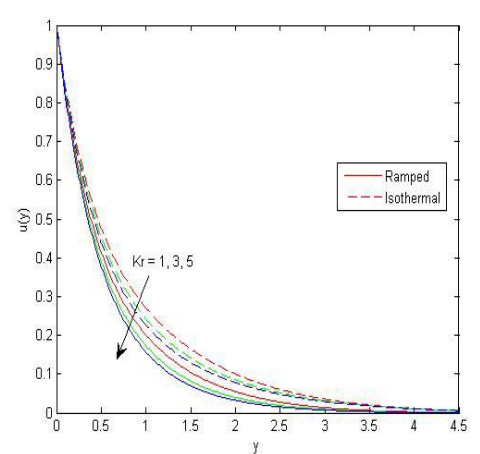

(a)

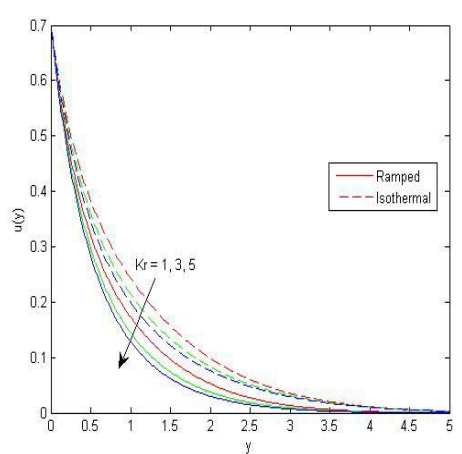

(b)

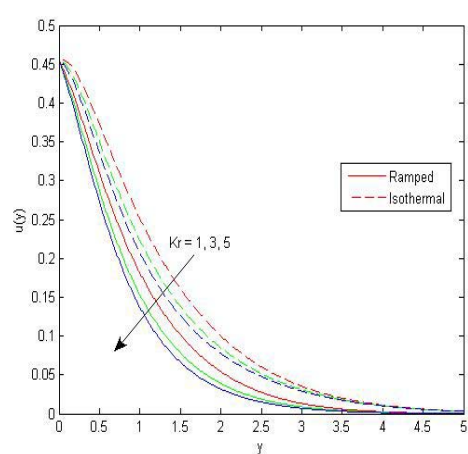

(c)

Fig.8. Effect of variation in the chemical reaction parameter $K r$ on the fluid velocity $u(y, t)$ when (a) $f(t)=H(t)$, (b) $f(t)=t H(t)$ and (c) $f(t)=\cos \omega t H(t)$ for $\mathrm{Sc}=0.6, \mathrm{Pr}=0.71, \mathrm{Gr}=2, G m=2, K=0.2, N=1$, $M=3, t=0.7$.

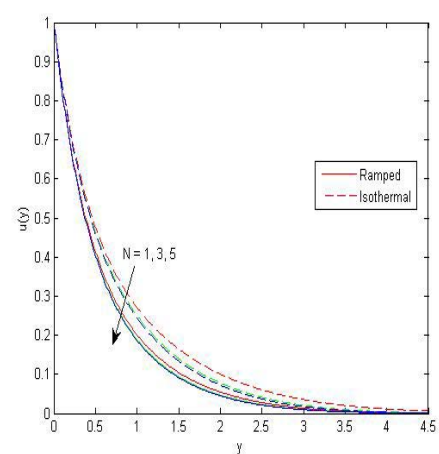

(a)

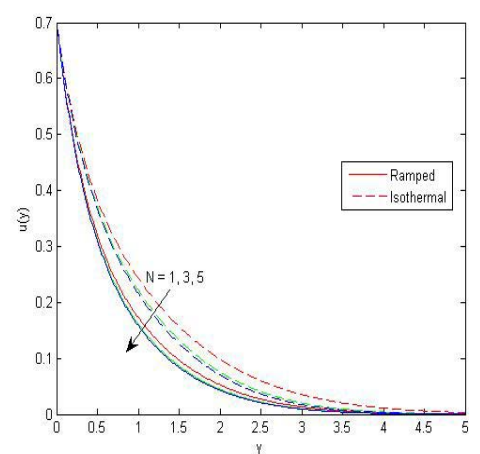

(b)

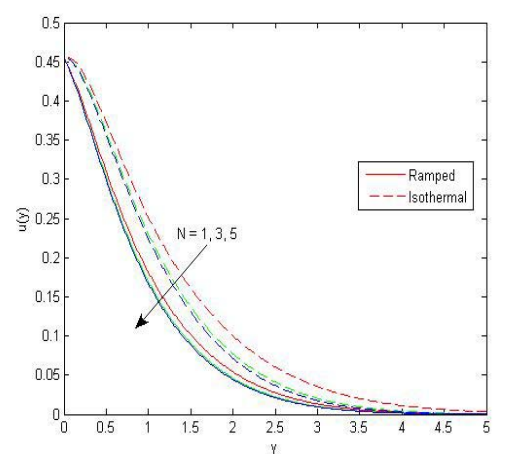

(c)

Fig.9. Effect of variation in the radiation parameter $N$ on the fluid velocity $u(y, t)$ when (a) $f(t)=H(t)$, (b) $f(t)=t H(t)$ and (c) $f(t)=\cos \omega t H(t)$ for $\mathrm{Sc}=0.6, \mathrm{Pr}=0.71, \mathrm{Gr}=2, G m=2, K=0.2, K r=1, M=3, t=0.7$. 


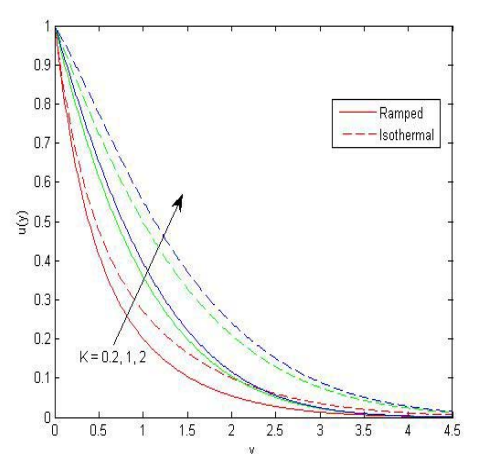

(a)

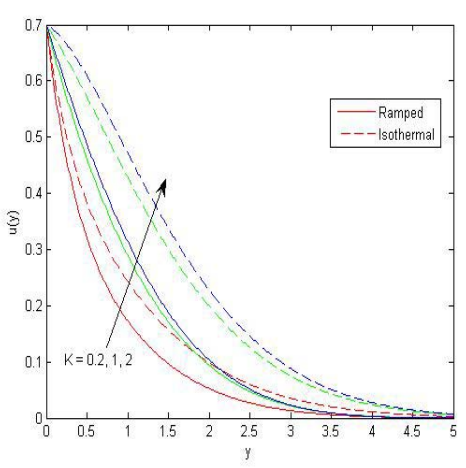

(b)

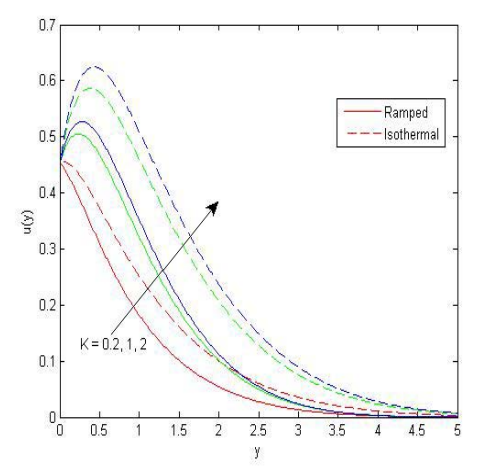

(c)

Fig.10. Effect of variation in the permeability parameter $K$ on the fluid velocity $u(y, t)$ when (a) $f(t)=H(t)$, (b) $f(t)=t H(t)$ and (c) $f(t)=\cos \omega t H(t)$ for $\mathrm{Sc}=0.6, \mathrm{Pr}=0.71, \mathrm{Gr}=2, G m=2, N=1, K r=1, M=3, t=0.7$.

Figure 11a shows that fluid temperature $\theta(y, t)$ increases on increasing time $t$. This implies that there is an enhancement in the fluid temperature with the progress of time throughout the thermal boundary layer region. It is evident from Fig. $11 \mathrm{~b}$ that the fluid temperature $\theta$ decreases on increasing Pr. An increase in the Prandtl number reduces the thermal boundary layer thickness. The Prandtl number signifies the ratio of momentum diffusivity to thermal diffusivity. It can be noticed that as Pr decreases, the thickness of the thermal boundary layer becomes greater than the thickness of the velocity boundary layer according to the well-known relation $\delta T / \delta \cong 1 / \operatorname{Pr}$ where $\delta T$ is the thickness of the thermal boundary layer and $\delta$ is the thickness of the velocity boundary layer, so the thickness of the thermal boundary layer increases as the Prandtl number decreases and hence the temperature profile decreases with an increase in the Prandtl number. In heat transfer problems, the Prandtl number controls the relative thickening of momentum and thermal boundary layers. When the Prandtl number is small, it means that heat diffuses quickly compared to the velocity (momentum), which means that for liquid metals the thickness of the thermal boundary layer is much bigger than the momentum boundary layer. Hence the Prandtl number can be used to increase the rate of cooling in conducting flows. Figure $11 \mathrm{c}$ is plotted to depict the influence of the thermal radiation parameter $N$ on the temperature profile. We observe in this figure that increasing the thermal radiation parameter produces a significant decrease in the thermal condition of the fluid flow for isothermal plates whereas it increases for ramped temperature. The effect of the heat absorption parameter $Q$, the Prandtl number Pr and time $t$ on the fluid temperature is in agreement with that of Nandkeolyar et al. [29].

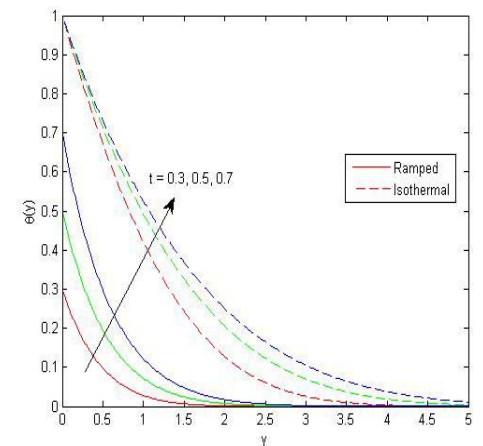

(a)

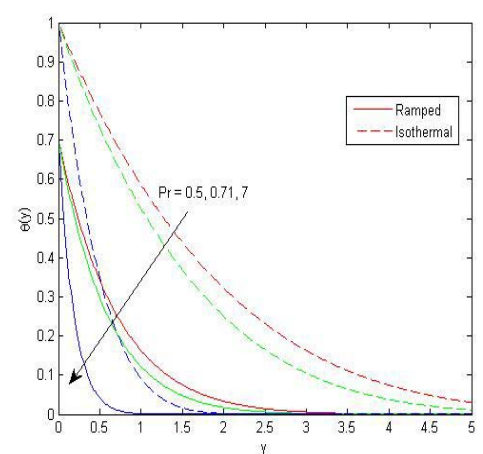

(b)

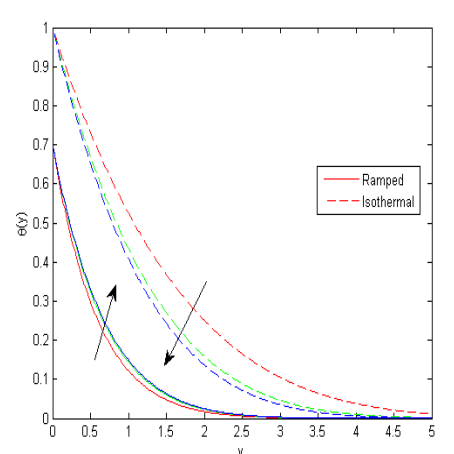

(c)

Fig.11. Effect of variation in (a) time $t$ (b) the Prandtl number Pr, and (c) the radiation parameter $N$ on $\theta(y, t)$. 
From Fig.12a, it is clear that with unabated mass diffusion into the fluid stream, the molar concentration of the mixture rises with increasing time and so there is an enhancement in species concentration with the progress of time throughout the boundary layer region. It is noticed from Fig. $12 \mathrm{~b}$ that the species concentration $\phi(y, t)$ decreases on increasing Sc. The Schmidt number embodies the ratio of the momentum to the mass diffusivity. The Schmidt number therefore quantifies the relative effectiveness of momentum and mass transport by diffusion in the concentration (species) boundary layer. As the Schmidt number increases the concentration decreases. This causes the concentration buoyancy effects to decrease. Figure 12c shows the influence of a chemical reaction on concentration profiles. In this study, we analyze the effects of a destructive chemical reaction $(\mathrm{Kr}>0)$. It is noticed that concentration distributions decrease when the chemical reaction increases. Physically, for a destructive case, chemical reaction takes place with many disturbances. This, in turn, causes high molecular motion, which results in an increase in the transport phenomenon, thereby reducing the concentration distributions in the fluid flow.

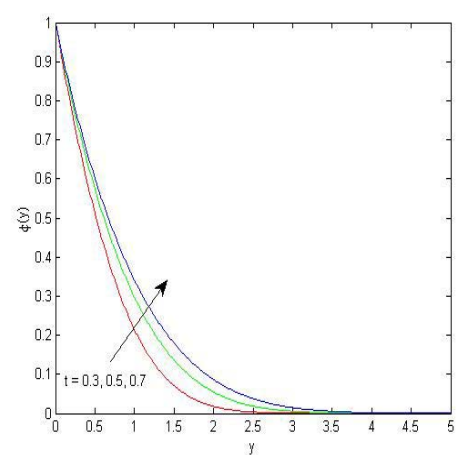

(a)

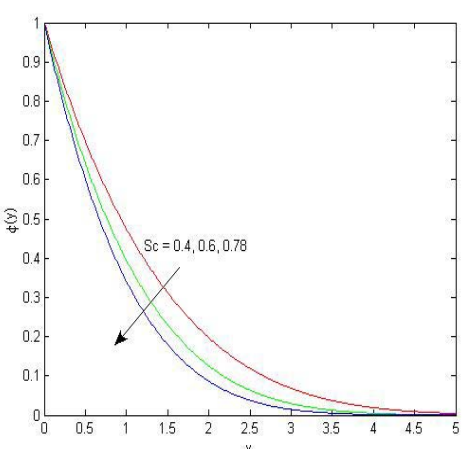

(b)

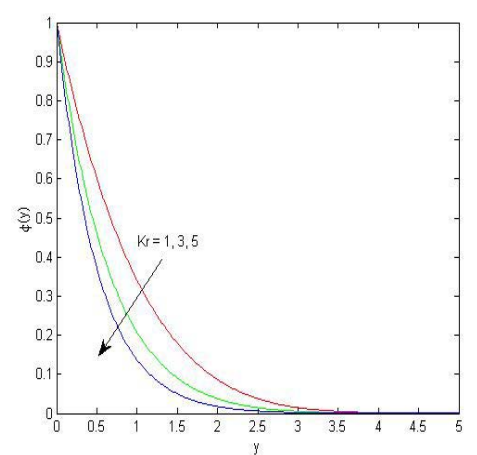

(c)

Fig.12. Effect of variation in (a) time $t$ (b) the Schmidt number Sc, and (c) the radiation parameter $N$ on $\phi(y, t)$.

The numerical values of the skin friction $\tau$ for a plate moving with uniform velocity, plate moving with uniform acceleration and plate moving with periodic acceleration for both ramped temperature and isothermal plates are displayed in a tabular form in Tabs 1-3, respectively, whereas the rate of heat transfer in terms of the Nusselt number $\mathrm{Nu}$ for both ramped temperature and isothermal plates and the rate of mass transfer in terms of the Sherwood number Sh are presented in Tab.4. It is noticed from Tabs 1-3 that the skin friction $\tau$ decreases with an increase in time $t$ except for the case when the plate moves with uniform velocity. In this case, the skin friction increases with an increase in time $t$ for both ramped temperature and isothermal plates. It is evident from the Tabs 1-3 that the skin friction for both ramped temperature and isothermal plates decreases with an increase in the value of $\operatorname{Pr}, Q, M, K r$ or $N$, whereas it increases with an increase in $\mathrm{Gr}, \mathrm{Gm}$ or $K$, which implies that, for both ramped temperature and isothermal plates, the heat absorption, magnetic field, chemical reaction or radiation tend to decrease the skin friction at the plate, while the thermal buoyancy force, mass buoyancy force and permeability of the medium have a reverse effect on it. It is noticed from Tab.4 that for both ramped temperature and isothermal plates the Nusselt number $\mathrm{Nu}$ increases with $\operatorname{Pr}, Q, N$ or $t$, which implies that the heat absorption, thermal radiation and time have a tendency to enhance the rate of heat transfer at the plate, whereas thermal diffusion has a reverse effect on it. The result for the Nusselt number $\mathrm{Nu}$ is in agreement with the results of Nandkeolyar et al. [29] for $\operatorname{Pr}, Q$ or time $t$. It is also evident from Tab.4 that the Sherwood number Sh decreases with an increase in time $t$, whereas it increases with an increase in Sc and $K r$. Thus both mass diffusivity and time $t$ tend to decrease the rate of mass transfer at the plate, whereas the chemical reaction parameter $K r$ increases the rate of mass transfer at the plate. 


\section{Conclusion}

The effects of chemical reaction and thermal radiation on an unsteady MHD free convection heat and mass transfer flow of a viscous, incompressible, electrically conducting and heat absorbing fluid past an infinite flat plate embedded in a porous medium were investigated. The flow was induced by a timedependent movement of the flat plate. Three cases of particular interest, namely (I) movement of the plate with uniform velocity (II) movement of the plate with uniform acceleration and (III) movement of the plate with periodic acceleration, were discussed. Exact solutions of the governing equations were obtained using the Laplace Transforms technique. A comprehensive set of graph for the fluid velocity, fluid temperature and species concentration is presented and the dependence on some physical parameters for both ramped temperature and isothermal plates is discussed. Significant findings are as follows:

For both ramped temperature and isothermal plates

- Heat absorption and magnetic field tend to retard the fluid flow, whereas the thermal buoyancy force, mass buoyancy force, thermal diffusion and permeability parameter have the opposite effect.

- Chemical reaction decelerates the fluid motion resulting in a decrease in the fluid velocity in the boundary layer.

- Thermal boundary layer thickness decreases with an increase in the radiation parameter and it results in decrease in the fluid velocity.

- Thermal diffusion and time accelerates the fluid temperature whereas the radiation parameter decreases the fluid temperature for isothermal plates.

- Mass diffusion and time tend to increase the species concentration, whereas the chemical reaction parameter has a reverse effect.

- Thermal diffusion, time, thermal buoyancy force, mass buoyancy force and permeability of the porous medium tend to increase the shear stress at the plate, whereas the heat absorption, magnetic field, chemical reaction parameter and thermal radiation parameter have a reverse effect.

- Heat absorption, thermal radiation and time tend to increase the rate of heat transfer, whereas thermal diffusion has the opposite effect.

- Chemical reaction parameter tends to increase the rate of mass transfer, whereas time and mass diffusion have the opposite effect.

\section{Nomenclature}

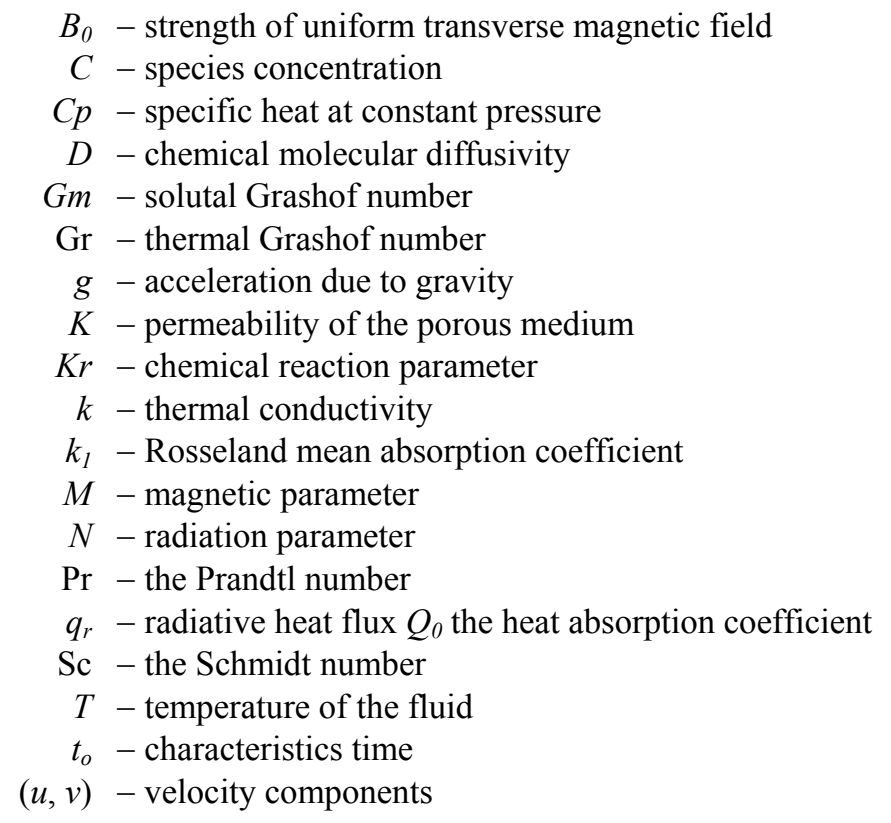


$(x, y)$ - transverse and normal directions

$\beta-$ volumetric coefficient of thermal expansion

$\beta^{\prime}-$ volumetric coefficient of expansion for concentration

$\sigma$ - electrical conductivity

$\sigma_{l}$ - Stefan-Boltzmann constant

$\rho$ - fluid density

$v$ - kinematic viscosity

$\theta$ - dimensionless temperature

$\phi$ - dimensionless concentration

\section{References}

[1] Nield D.A. and Bejan A. (1998): Convection in porous media, 2nd Edition. - Berlin: Springer-Verlag.

[2] Hiremath P.S. and Patil P.M. (1993): Free convection effects on oscillatory flow of couple stress field through a porous medium. - ACTA Mechanica, vol.98, No.1-4, pp.143-158.

[3] Muthucumaraswamy R. and Valli-Ammal V. (2010): The theoretical study of unsteady flow past an exponentially accelerated infinite isothermal vertical plate with variable mass diffusion in the presence of homogeneous chemical reaction of first order. - Theo. Appl. Mech., vol.37, No.4, pp.251-262.

[4] Das U.N., Deka R. and Soungalgekar V.M. (1994): Effects on mass transfer on flow past an impulsively started infinite vertical plate with constant heat flux and chemical reaction. - Forschungim Ingenieurwesen, vol.60, pp.284-287.

[5] Suneetha S., Bhaskar Reddy N. and Ramachandra Prasad V. (2009): Effects of thermal radiation on the natural convective heat and mass transfer of a viscous incompressible gray absorbing-emitting fluid flowing past an impulsively started moving vertical plate with viscous dissipation. - Thermal Science, vol.13, No.2, pp.71-181.

[6] Satter M.A. and Kalim H. (1996): Unsteady free-convection interaction with thermal radiation in a boundary layer flow past a vertical porous plate. - J. Math. Phys. Sci., vol.30, No.1, pp.25-37.

[7] Aydin O. and Kaya A. (2008): Radiation effect on MHD mixed convection flow about a permeable vertical plate. Heat Mass Transfer, vol.45, pp.239-246.

[8] Arpaci V.S. (1968): Effects of thermal radiation on the laminar free convection from a heated vertical plate. - Int. J. Heat Mass Transfer, vol.11, pp.871-881.

[9] Cess R.D. (1966): Interaction of thermal radiation with free convection heat transfer. - Int. J. Heat Mass Transfer, vol.9, pp.1269-1277.

[10] Cheng E.H. and Ozisik M.N. (1972): Radiation with free convection in an absorbing emitting and scattering medium. - Int. J. Heat Mass Transfer, vol.15, pp.1243-1252.

[11] Raptis A. (1998): Radiation and free convection flow through a porous medium. - Int. Comm. Heat Mass Transfer, vol.25, No.2, pp.289-295.

[12] Hossain M.A. and Takhar H.S. (1996): Radiation effects on mixed convection along a vertical plate with uniform surface temperature. - Heat Mass Transfer, vol.31, pp.243-248.

[13] Hossain M.A. and Takhar H.S. (1999): Thermal radiation effects on the natural convection flow over an isothermal horizontal plate. - Heat Mass Transfer, vol.35, pp.321-326.

[14] Das U.N., Deka R. and Soungalgekar V.M. (1996): Radiation effects on flow past an impulsively started vertical plate-an exact solution. - J. Theo. Appl. Fluid Mech., vol.1, No.2, pp.111-115.

[15] Ahmed T. and Alam Md Mahmud (2013): Finite difference solution of MHD mixed convection flow with heat generation and chemical reaction. - Proc. Eng., vol.56, pp.149-156.

[16] Seth G.S., Sarkar S. and Hussain S.M. (2014): Effects of Hall current, radiation and rotation on natural convection heat and mass transfer flow past a moving vertical plate. - Ain Shams Eng. Journal, vol.5, No.2, pp.489-503. 
[17] Cookey C.I., Ogulu A. and Omubo Pepple V.B. (2003): Influence of viscous dissipation and radiation on unsteady MHD free convection flow past an infinite heated vertical plate in a porous medium with time-dependent suction. Int. J. Heat Mass Transfer, vol.46, pp.2305-2311.

[18] Suneetha S., Bhaskar Reddy N. and Ramachandra Prasad V. (2008): Thermal radiation effects on MHD free convection flow past an impulsively started vertical plate with variable surface temperature and concentration. - J. Naval Archit. Marine Eng., vol.5, No.2, pp.57-70.

[19] Mahmoud M.A.A. (2009): Thermal radiation effect on unsteady MHD free convection flow past a vertical plate with temperature dependent viscosity. - Can J. Chem. Eng., vol.87, pp.47-52.

[20] Seth G.S., Nandkeolyar R. and Ansari Mds. (2013): Effects of thermal radiation and rotation on unsteady hydromagnetic free convection flow past an impulsively moving vertical plate with ramped temperature in a porous medium. - J. Appl. Fluid Mech., vol.6, pp.27-38.

[21] Huges W.F. and Yong F.J. (1966): The electro-Magneto-Dynamics of fluids. - New York, USA: John Wiley \& Sons.

[22] Sacheti N.C., Chandraan P. and Singh A.K. (1994): Exact solution for unsteady MHD free convection flow on an impulsively started vertical plate with constant heat flux. - Int. Comm., Heat Mass Transfer, vol.21, No.1, pp.131142.

[23] Takhar H.S., Gorla R.S.R. and Soundalgekar V.M. (1996): Effect of radiation on MHD free convection flow past a semi-infinite vertical plate using Runge-Kutta-Merson quadrature. - Int. J. Numerical Methods for Heat \& Fluid Flow, vol.6, pp.77-83.

[24] Abd-El-Naby M.A., Elasayed M.E., Elbarbary N.Y. and Abdelzem (2003): Effects of radiation on MHD unsteady free convection flow over a vertical plate with variable surface temperature. - J. Appl. Math, vol.2, pp.65-86.

[25] Kishore P.M., Prasad Rao N.V.R.V., Varma S.V.K. and Venkataramana S. (2013): The effects of radiation and chemical reaction on unsteady MHD free convection flow of viscous fluid past an exponentially accelerated vertical plate. - Int. J. of Physical and Mathematical Sci., vol.4, No.1, pp.300-317.

[26] El-Fayez F.M.N. (2012): Effects of chemical reaction on the unsteady free convection flow past an infinite vertical permeable moving plate with variable temperature. - J. of Surface Engineered Materials and Adv. Tech., vol.2, pp.100-109.

[27] Sekhar D.V. and Reddy G.V. (2012): Effects of chemical reaction on MHD free convective oscillatory flow past a porous plate with viscous dissipation and heat sink. - Adv. in Appl. Sci. Res., vol.3, No.5, pp.3206-3215.

[28] Shateyi S. and Marewo G.T. (2014): Numerical analysis of unsteady MHD flow near a stagnation point of a twodimensional porous body with heat and mass transfer, thermal radiation and chemical reaction. - Boundary Value Problems, 2014:218.

[29] Nandkeolyar R., Das M. and Sibanda P. (2013): Exact solutions of unsteady MHD free convection in a heat absorbing fluid flow past a flat plate with ramped wall temperature. - Boundary Value Problems, vol.1, pp.1-16.

[30] Cramer K. and Pai S. (1973): Magnetofluiddynamics for Engineers and Applied Physicists. - New York: McGrawHill.

[31] Meyer R.C. (1958): On reducing aerodynamic heat-transfer rates by magnetohydrodynamic techniques. - J. Aeronaut. Sci., vol.25, pp.561-572.

Received: October 6, 2016

Revised: September 18, 2017 